\title{
Joint Latency and Reliability-Aware Controller Placement
}

\author{
Kurdman Abdulrahman Rasol Rasol \\ Department of Computer Architecture \\ Universitat Politècnica de Catalunya (UPC) \\ Barcelona, Catalonia, Spain \\ kurdman.rasol@upc.edu
}

\author{
Prof. Jordi Domingo-Pascual \\ Department of Computer Architecture \\ Universitat Politècnica de Catalunya (UPC) \\ Barcelona, Catalonia, Spain \\ jordi.domingo@ac.upc.edu
}

\begin{abstract}
In network architectures based on Software Defined Networking (SDN) the control plane (control logic) is separated from the network data plane (forwarding plane) while traditional network routers combine both. Software Defined networks facilitates a centralized networking system where a logical controller manages the global view of the network. In this paper, we first propose a new metric on the controller placement problem (CPP) that simultaneously considers the communication latency and communication reliability both between switches and controllers and between controllers. Reliability is considered for singlelink failure. We model the problem of determining the optimal controller placement to provide low latencies in the control plane traffic. The objective of this study is to minimize the average accumulated latency by jointly taking into account the latency between controller to switches and inter-controller while optimizing their placement for achieving an optimal balance simultaneously. The optimization problem is formulated as a mixed-integer linear programming (MILP) model under the constraints of latency and reliability. We evaluated the performance of our proposed metric by using the Internet2 OS3E network topology. Different from previous work, we focus on the control traffic exchanged among controllers to synchronize their shared data structure. Results demonstrate that the proposed method is promising.

Index Terms-Software Defined Networking, Control Plane Latency, Multiple Controller Placements, Single-Link Failure, Backup Path, Mixed-Integer Linear Programming
\end{abstract}

\section{INTRODUCTION}

Unlike traditional networking technologies, which integrates both the data plane and control plane on the same networking device, Software Defined Networks (SDNs) are characterized by disassociating a network architecture in the data plane (forwarding plane) and the control plane (controller logic). SDN provides a logically centralized control plane intelligence to manage the whole network and network programmability [1], [2]. The forwarding/data plane is responsible for the forwarding devices in network traffic, whereas the data plane is programmed and managed by the control plane. The control plane is responsible and capable of handling the network traffic, generating rules and policies to make high-level decisionmaking and configuration for the forwarding devices (i.e., switches and routers) with the help of the controllers. The data packets and control packets share the same network infrastructure.

In SDNs, deploying a single controller for network management makes the network presents reliability issues. Multiple controllers are deployed to improve network performance and manage the entire network more efficiently and flexibly.

In a SDN control plane, two functional operations are identified. First, the control traffic exchanged between controllers and switches attached to the controller, referred to as $(\mathrm{Ctr}-\mathrm{Sw})$, to configure, manage, and monitor the switches. Second, the control traffic exchanged among controllers (or inter-controller communication), indicated as (Ctr-Ctr), which supports the interaction between controllers (i.e., the control traffic to keep the shared data structures synchronized). The control plane is a combination of inter-controller and switches to controller communications. The ( $\mathrm{Ctr}-\mathrm{Ctr})$ communication of SDN domains is established utilizing east/westbound interfaces. The southbound interface is responsible for (Ctr-Sw) communication in SDN domain (i.e., OpenFlow). Thus, the (Ctr-Ctr) latencies must be considered along with the (Ctr-Sw) latencies when evaluating the control plane performance. As a result, the optimal controller placement in a given network should consider both kinds of latencies.

However, most of the related literature only focuses on the latencies of the controllers to switches interactions, while neglecting the latency introduced by the controller-to-controller communications. In this paper, we focus on the controller placement problem by considering the impact of both latencies. The balance between these two types of traffic (Ctr-Sw) and ( $\mathrm{Ctr}-\mathrm{Ctr})$ is considered in the proposed solution.

The Ctr-Ctr traffic increases when multiple controllers are deployed, while few controllers increase the $\mathrm{Ctr}-\mathrm{Sw}$ traffic latency. The Ctr-Ctr traffic is important to maintain the shared view of the network state and information providing a global view to run network applications correctly and programming abstractions. Alternatively, the Sw-Ctr traffic essentially depends on the network application running on the controller. Usually, the south-bound interface (Ctr-Sw traffic) interactions are more responsive and quicker than the east-west interface (Ctr-Ctr traffic) interactions. Depending on the application of the control plane, it is more significant to minimize the $\mathrm{Ctr}-\mathrm{Ctr}$ latencies than $\mathrm{Ctr}-\mathrm{Sw}$ traffic interactions. This is a management and deployment decision that may impact the overall performance.

The take-home message of this paper is that for each network application running on the control plane, we can 


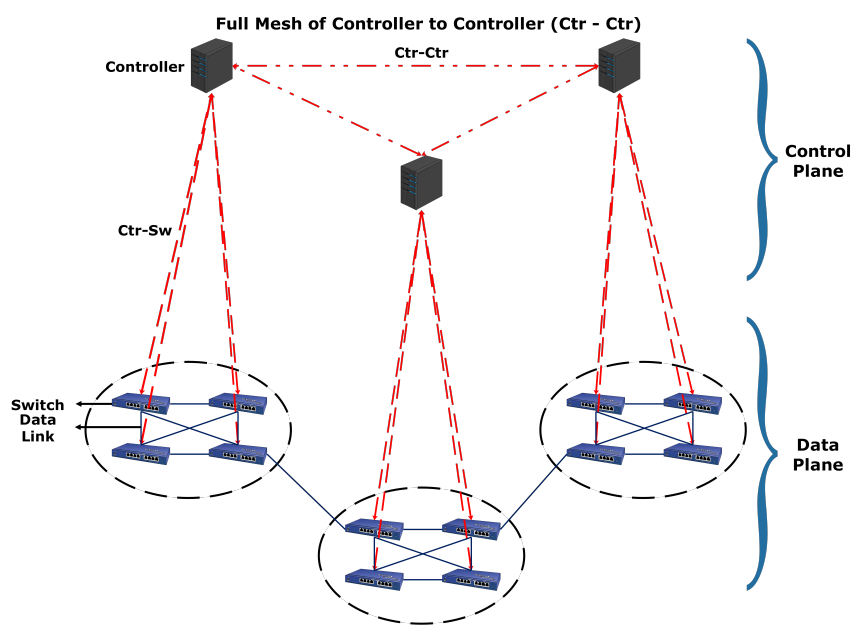

Fig. 1. The Architecture of SDN Control Plane

choose the weights between $\mathrm{Ctr}-\mathrm{Sw}$ and $\mathrm{Ctr}-\mathrm{Ctr}$ latencies to find the best solution. This paper considers two main objectives; firstly, is to minimize the accumulated control plane latency, which takes into consideration both latencies from the Ctr-Sw and Ctr-Ctr at the same time. Secondly, to include reliability considering the effect of link failures on the control traffic Ctr-Sw and Ctr-Ctr.

The rest of the paper organized as follows. Section II gives a description of related work in the Controller Placement Problem (CPP). We formulate the problem mathematically in Section III. Section IV presents our results in the control plane. Finally, this paper is concluded in Section V.

\section{RELATED WORK}

In this section, we show a brief review of the controller placement problem in SDN. The CPP is a significant research problem in a pure SDN. This problem has formally proved that CPP is similar to the facility location problem and is NP-Hard [3]. The controller placement strategies took into account only the latency between the controllers and their assigned switches. The authors evaluated the performance of the CPP on the Internet 2 OS3E topology [4]. Heller et al is designed to find the minimum number of controllers required in the network and where place each controller to provide low latencies between switches and their associated controller [5]. An optimized K-means algorithm is designed to address the problem for latency minimization between switches and controllers [6]. On one hand, K-center is proposed to address this problem for minimizing the maximum latency between the controller and switches [7].

In controller placements, reliability is also a key problem to improve the network performance of the network. Yuqi Fan et al. [8] proposed a new latency metric Latency-Aware Reliable Controller placement algorithm (LARC) for the problem that focuses on the latency only between the switches and their assigned controller's communication with a single-link failure and proposed an efficient algorithm for the problem. The objective to minimize the average accumulated latency on both primary and backup paths are jointly considered during the placement of controllers. But it does not consider the communication among controllers.

In [9], the authors propose an efficient Reliability-aware Controller Placement (RCP) algorithm against the state-ofart: Latency-Aware Reliable Controller placement algorithm (LARC) [8] and the optimized K-means network partition algorithm (OKMP) [6]. The RCP algorithm divides the network into multiple sub-networks and places a controller in each sub-network. A local search algorithm is presented to determine controller placements and switch-to-controller mapping relationship. The simulation results showed that the RCP algorithm provides better performance to reduce the backup path latency and the accumulated latency of the primary and backup paths. But, controller-to-controller communication has been ignored.

AK Singh et al. [10] focus on the reliable controller placement problem (RCPP) and use Varna-based optimization (VBO) for a reliable CPP that minimizes the total average latency of the network. Results show that the proposed algorithm provides better performance compared with other optimization methods, i.e., particle swarm optimization (PSO) [11], teacher learning-based optimization (TLBO) [12], and Jaya algorithms [13] based solution for RCPP.

K. S. Sahoo et al. [14] propose Particle Swarm Optimization (PSO) and Firefly (FFA) for solving controller placement problem in SDN-based wide area networks. The objective of their work is to minimize the latency to find the optimal number and location of the controller, which take into account the communication between the switch to controller and controller to controller. Their results show that the FFA achieves better performance than PSO and the time taken by both are in almost close level.

In our study, we investigate a new metric for Joint Latency and Reliability-aware Controller Placement Optimization (LRCP), which takes into account both the communication reliability and the communication latency between the controller and their associated switches as well as the controllerto-controller simultaneously. Reliability considers a single-link failure. Future studies will to address controller placement including capacity constraints.

\section{OPTIMIZATION MODEL}

An SDN network is represented as a graph $G=(V, E)$, where switches (or nodes) set $V$ consists of SDN-enabled network devices and edges (or links) set $E$ includes the communication links among devices, where the link weight indicates the distance between two nodes. The set of switches $V$ and edges $E$ are defined in (1) and (2):

$$
\begin{gathered}
V=\left\{v_{i} \mid i=1,2, \ldots, n\right\} \\
E=\left\{\operatorname{link}\left(v_{i}, v_{j}\right) \mid v_{i}, v_{j} \subset V\right\}
\end{gathered}
$$


TABLE I

NOTATIONS AND DEFINITIONS.

\begin{tabular}{|c|c|}
\hline NOTATIONS & DEFINITION \\
\hline$G(V, E)$ & Graph $\mathrm{G}$, where $\mathrm{V}$ is a set of switches and $\mathrm{E}$ is set of edges between switches \\
\hline$V$ & Set of network devices (Switches or nodes) in the network \\
\hline$E$ & Set of physical links (or edges) among network devices/switches \\
\hline$C$ & Controller set, where $(C \subset V)$ \\
\hline$M$ & A matrix consisting of the shortest path latency. For each index $(i, j)$, it corresponds to the shortest path latency from node $i$ to node $j$. \\
\hline$i, j$ & Switches or nodes $i$ and $j$ \\
\hline$(i, j)$ & The link from between nodes $i$ and $j$ \\
\hline $\operatorname{dist}_{(i, j)}$ & The distance between nodes $i$ and $j$ \\
\hline$n$ & Total number of nodes in the network, where $(n=|V|)$ \\
\hline$k$ & Total number of controllers to be installed in the network, where $(k \leq n)$ \\
\hline$p_{i, k}$ & The link set of the primary path between switch $i$ and controller $k$ \\
\hline$l_{i, k}^{p}$ & The latency of the primary path between switch $i$ and controller $k$ \\
\hline$l_{i, k}^{b}$ & The average latency of the backup paths between switch $i$ and controller $k$ \\
\hline$l_{i, k, i^{\prime}, j^{\prime}}^{b}$ & The latency of the backup path between switch $i$ and controller $k$ under the link failure $\left(i^{\prime}, j^{\prime}\right)$. \\
\hline$L A R C(i, k)$ & The accumulated latency in the primary path and backup paths between switch $i$ and controller $k$ \\
\hline
\end{tabular}

Where, $v_{i}$ and $v_{j}$ are switch nodes, link $\left(v_{i}, v_{j}\right)$ is the link between nodes $v_{i}$ and $v_{j}$ and $n$ denotes the total amount of nodes $(n=|V|)$.

$C$ represents the set of controller nodes in the network topology, and $E_{C}$ represents the set of paths between the controllers, which are shown in (3) and (4):

$$
\begin{gathered}
C=\left\{c_{i} \mid i=1,2, \ldots, k\right\} \\
E_{C}=\left\{\operatorname{link}\left(c_{i}, c_{j}\right) \mid c_{i}, c_{j} \subset C\right\}
\end{gathered}
$$

Where, $c_{i}$ and $c_{j}$ are controller nodes, link $\left(c_{i}, c_{j}\right)$ is the link between nodes $c_{i}$ and $c j$ and $k$ is the number of controllers to be deployed throughout the network $(k \leq n)$.

$M$ represents the distance matrix between all nodes and $\operatorname{dist}_{(i, j)}$ represents the distance between nodes $i$ and node $j$. A node may be a simple switch node or a controller node which is placed on the switch node. As an example, in Figure 1 there are 12 nodes from switches to 3 are controllers, that is $n=12$ and $k=3$. The details of the notation in this paper are summarized in Table I.

We propose a new metric called Joint Latency and Reliability-aware Controller Placement Optimization (LRCP) based on the original Controller Placement Problem (CPP) model [5] with some additional constraint and terms for the objective function. As a first approach, a metric is proposed to find the trade-off between the controller and their associated switches latency (Ctr-Sw) and inter-controller latency (Ctr-Ctr) at the same time. The goal of this metric is to minimize the accumulated latency of the network, mathematically formulated as described in (5):

$$
\text { lat }=\min \left(\lambda z_{1}+(1-\lambda) z_{2}\right)
$$

The objective function in the first part of the model formulation considers the latency between switches and their assigned controller ( $z 1$ weighted by $\lambda$ ) and the second part of the function considers the controller-to-controller latency $(z 2$ weighted by $(1-\lambda))$. On the other hand, the accumulated latency is the weighted combination both latency between Ctr$\mathrm{Sw}$ and Ctr-Ctr communication. In [15], we present a detailed evaluation of this metric and its applicability.

The goal of the model is to determine the optimal minimum accumulated latency placements and controller locations to achieve a given balance between switches to the assigned controller latencies, and inter-controllers latencies in the control plane. The latency between two nodes is measured as the distance by using Dijkstra's algorithm to calculate the shortest path distance between pairs of nodes in the network. According to this model, each controller is placed with a switch and a single controller controls each switch.

The new metric, LRCP, is built as an extension of [15], including reliability for single-link failures, following the work presented in LARC [8]. LARC $(i, j)$ represents the shortest distance between switch node $i$ to controller node $j$, $n$ represents the total amount of nodes and $k$ represents the number of controllers.

1) The average switch to controller latency $(C t r-S w)$ : The average latency between controllers and switches is calculated as shown in (6):

$$
z_{1}=\frac{\sum_{i \in V, j \in C} \operatorname{LARC}_{(i, j)} x_{i, j}}{(n-k)}
$$

This optimization problem is known as the minimum K-means clustering algorithm problem.

2) The average inter-controller latency $(C t r-C t r)$ : The average latency is shown in (7)

$$
z_{2}=\frac{\sum_{i \in V, j \in C} \operatorname{LARC}_{(i, j)} x_{i, i} y_{j, j}}{n_{(C t r-C t r)}}
$$

The number of possible paths between controller-controller is dependent on number of controllers $k$ and is (8):

$$
n_{(C t r-C t r)}=k(k-1) / 2
$$




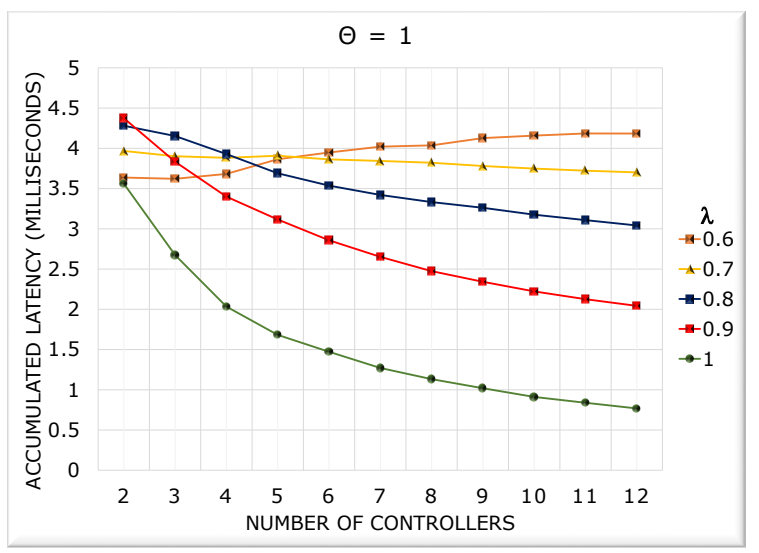

Fig. 2. The average accumulated latency (milliseconds) using primary path only $(\theta=1)$

We assume that there is not more than one link failure (singlelink failure).

$$
\mathrm{LARC}=\theta l^{p}+(1-\theta) l^{b}
$$

Equation (9) represents the LARC latency includes both the average latency of the primary path plus an average of the alternative backup paths that may be used when one singlelink of the primary path fails. The LARC latency is used for both $\mathrm{Ctr}-\mathrm{Sw}$ and $\mathrm{Ctr}-\mathrm{Ctr}$ paths. The average latency of the primary paths is computed as shown in Equation (10).

$$
l^{p}=\frac{\sum_{i \in V, k \in C} l_{i, k}^{p} \cdot x_{i, k}}{n}
$$

In case of a single-link failure of the primary path $l_{i, k}^{p}$, the backup path needs to be built and updated to replace the failed primary path in the network.

$$
\begin{aligned}
l_{i, k}^{b} & =\frac{\sum_{\left(i^{\prime}, j^{\prime}\right) \in p_{i, k}} l_{i, k, i^{\prime}, j^{\prime}}^{b}}{\left|p_{i, k}\right|} \\
l^{b} & =\frac{\sum_{i \in V, k \in C} l_{i, k}^{b} \cdot x_{i, k}}{n}
\end{aligned}
$$

When a single-link failure occurs $\left(i^{\prime}, j^{\prime}\right)$ in the primary path $l_{i, k}^{p}$, the backup path needs to set up to connect switch $i$ and its assigned controller $k$. We apply Dijkstra's algorithm to find the shortest path which avoids the failed link to rebuild the connection between switch $i$ and controller $k$. Since every link on primary path $l_{i, k}^{p}$ may fail, we compute the average latency of the backup path between switch $i$ and controller $k$. By considering all single-link failures in the network, the average latency of all the backup paths in the network is computed by Equation (11) and Equation (12).

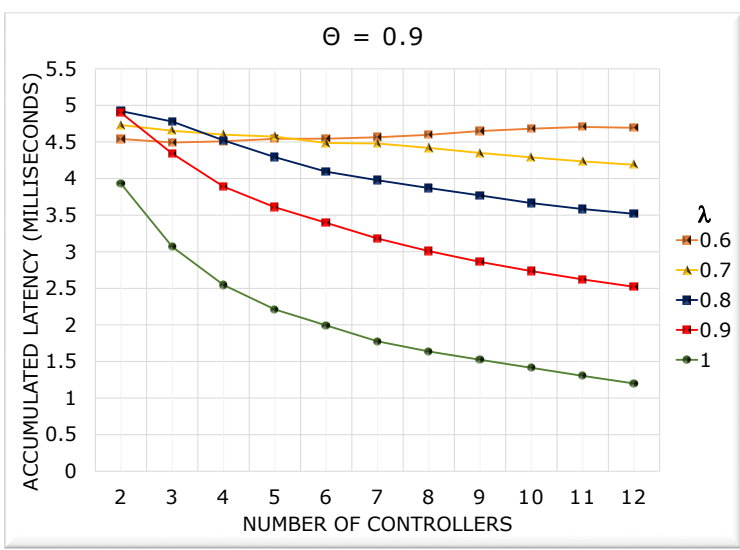

Fig. 3. The average accumulated latency (milliseconds) when $\theta=0.9$

This model is formulated under the following constraints:

$$
\begin{aligned}
& \sum_{j \in C} x_{i, j}=1, \forall i \in V \\
& \sum_{i \in V} y_{i, j}=1, \forall j \in C \\
& y_{i, j} \leq x_{i, j}, \forall i \in V, \forall j \in C \\
& x_{i, j}, y_{i, j} \in\{0,1\}
\end{aligned}
$$

These constraints ensure that: each switch is assigned to exactly one controller (13); each controller is located onto exactly one switch (14); a switch $i$ is mapped to the controller $j$ if controller $j$ is placed onto switch $i$ (15) and Constraint (16) guarantees that $x_{i, j}$ and $y_{i, j}$ are binary integer variables (equal to 0 or 1). Binary decision variables are described as in (17) and (18):

$$
\begin{aligned}
& x_{i, j}= \begin{cases}1, & \text { if switch } i \text { is mapped to controller } j . \\
0, & \text { otherwise. }\end{cases} \\
& y_{i, j}= \begin{cases}1, & \text { if controller } k \text { is placed onto switch } i . \\
0, & \text { otherwise. }\end{cases}
\end{aligned}
$$

Using LARC distance, we build a topology graph where the weights of the links include an extra value representing the average backup paths latency. The optimization using this topology aims to determine the best placement of a set of controllers when a single-link failure occurs.

\section{EVALUATION RESULTS}

We aim to minimize the total accumulated average latency taking into consideration the latency between switches and their assigned controller as well as the latency among controllers simultaneously with a single-link failure. The real network topology used is Internet2 OS3E network (34 nodes and 42 links). Mathematical modelling is written in Python. The optimization program uses Gurobi Optimizer to solve Mixed Integer Linear Programming (MILP) model. 
In our model, we have two different types of weights, $\lambda$ and $\theta$. The value of the $\lambda$ is utilised for the weights between $\mathrm{Ctr}-\mathrm{Sw}$ and Ctr-Ctr latencies. Depending on the considered control plane application, more weight for minimizing Ctr-Sw traffic or Ctr-Ctr traffic may be required. On the other hand, the value of the $\theta$ is used for the weights of the latency of the primary path $l^{p}$ and the average latency of the backup path $l^{b}$. Therefore, the controller location may vary according to both parameters $\lambda$ or $\theta$.

When $\theta=1$ and $\lambda=1$, the results depend on the average latency between switches and their assigned controllers only and considers the primary paths only. This case corresponds with the basic CPP and LARC optimization using the primary paths only [5], [8]. Average latency decreases when more controllers are used. As expected, we obtain the same results as CPP and LARC, that is, the same controller placements and the set of switches per controller, and same optimal controller to switches latency.

The case when $\theta=1$ with different values of $\lambda$ corresponds with the basic joint placement latency optimization (LCP) [15]. Figure 2 depicts the comparison results of average accumulated latency and the impact of $\lambda$ on latency when the number of controllers varied from 2 to 12 . It is interesting to note that, as the number of controllers deployed reaches a certain value in the network, the average accumulated latency begins to increase. By increasing the number of controllers, the latency among controllers increases. As the value of $\lambda$ grows, the average accumulated latency increases.

The reliability of the network decreases as the failure probability increases. Therefore, controller placements influence on both network latency and reliability in the whole network.

Figure 3 presents the corresponding results when $\theta=0.9$. That is, the accumulated latency takes into account the average of backup paths and this implies slightly different controller placements and associations with their corresponding switches. As an example, for five controllers $(k=5)$ and $\lambda=0.8$ the accumulated latency considering the primary paths is $3.69 \mathrm{~ms}$ (Fig. 2) while considering the cost of a link failure with $\theta=$ 0.9 the new placement gives an accumulated latency of 4.29 ms (Fig. 3).

Figure 4 shows how different values of $\theta$ influence the placement of a set of controllers and the distribution of switches attached to each controller. For five controllers $(k$ =5), Fig. 4 (a) corresponds to (Fig. 2) when $\theta=1$ and $\lambda=$ 0.8 while Fig. 4 (b) corresponds to (Fig.3) when $\theta=0.9$ and $\lambda=0.8$.

For a given probability of a link failure, the sub-optimal placement that considers this event and corresponding backup paths provide a slightly larger value of the accumulated latency respect value for the primary paths only.

Figure 5 analyzes the effect of the number of controllers on the average accumulated latency between switch and controller $(\lambda=1)$. These results are the same as the original LARC proposal [8]. We observe that by increasing $\theta$, the average accumulated latency decreases. Remind that $\theta=1$ means we are using the primary paths only.

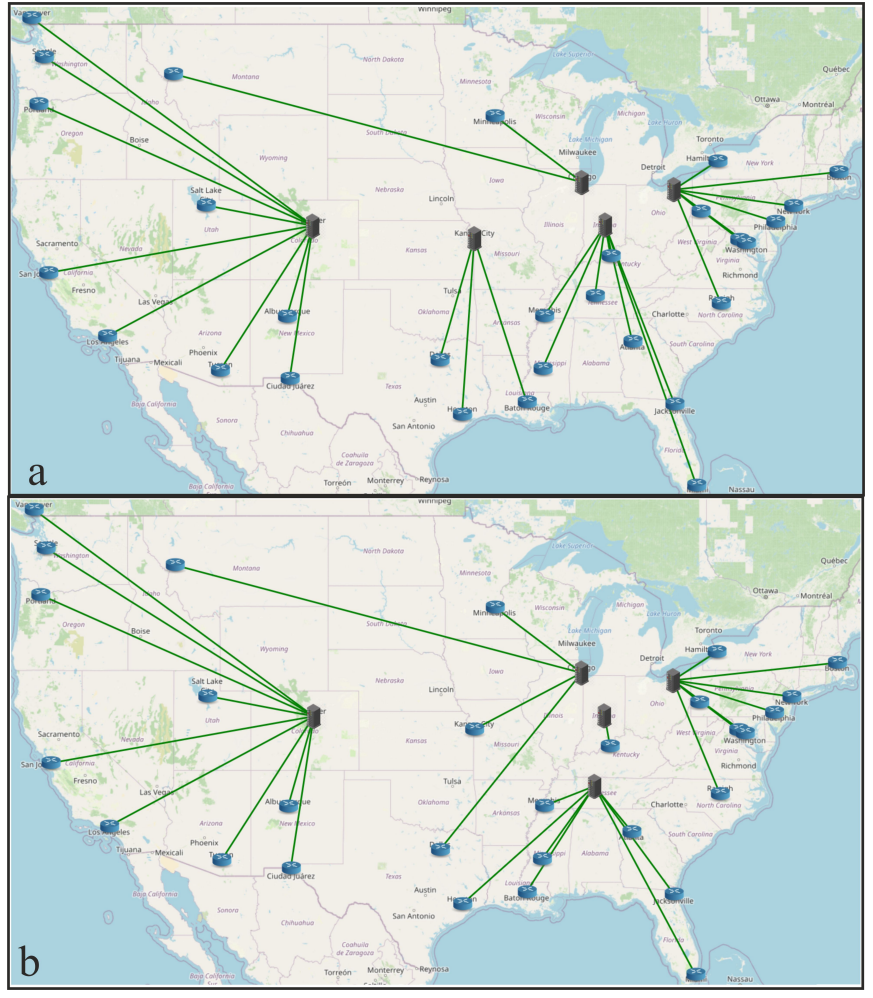

Fig. 4. The optimal controller location and their associated switches a) $\theta=$ 1 and b) $\theta=0.9$

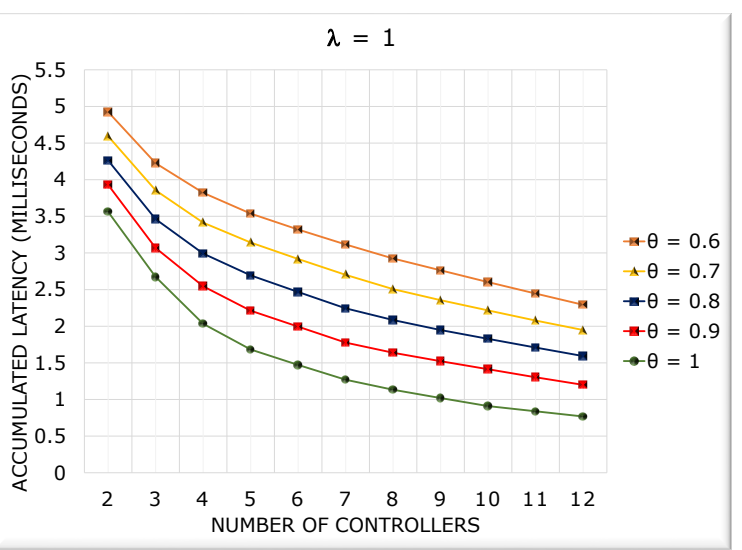

Fig. 5. The average accumulated latency (milliseconds) for switch controller $(\lambda=1)$

Figure 6 shows the average accumulated latency of our proposal (LRCP) for a particular case when the number of controllers is five $(k=5)$. For $\theta=1$, LCP [15] and LRCP are the same. In particular, the case when $\lambda=1$ corresponds to the previous work of CPP and LARC for $\theta=1$. CPP places the controllers to optimize the primary path for the Ctr-Sw latency only without considering link failures [5]. LARC deploys the controllers intending to minimize the average latency of primary and backup paths between controllers and their 


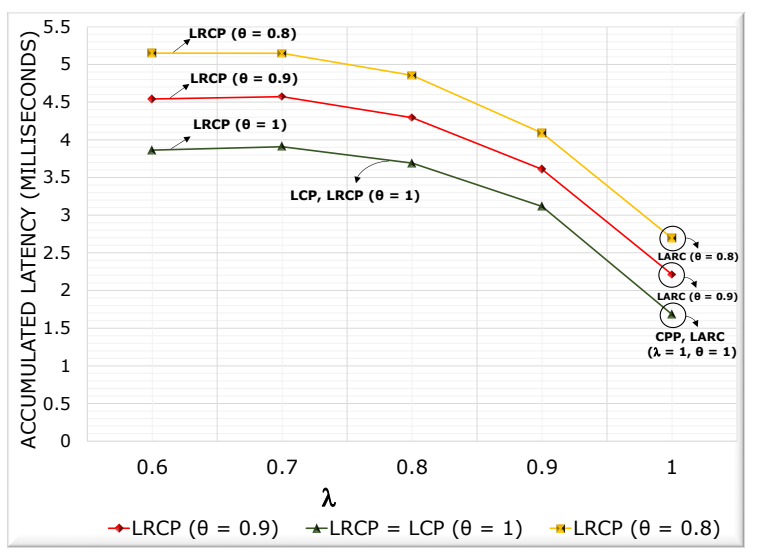

Fig. 6. The average accumulated latency (milliseconds) of LRCP for 5 controllers $(k=5)$

associated switches (Ctr-Sw) when a single link failure occurs. However, controller-to-controller latency is not considered in LARC [8]. The average latency increases while the weights of backup path latency growth ( $\theta$ decreases) because the average latency of the backup path is larger than the average latency of the primary path.

\section{CONClusion}

A single controller is not capable to manage huge traffic and may not be able to handle the scalability and reliability issues in the network. Placing multiple controllers is an essential requirement to improve network performance and reliability. We tackle the original controller placement problem (CPP) extending it and considering the communication latency and communication reliability. We conduct experiments using Internet2 OS3E network topology, so that we may compare our results with the previous works. Our proposed metric LRCP is built as an extension of the CPP and LCP models including reliability for single-link failures, following the work presented in the LARC model. The latency among controllers increases as the number of controllers becomes larger. When the number of controllers decreases, the latency between controller to switch traffic increases.

The two parameters of the model ( $\lambda$ and $\theta$ ) play a key role in the deployment of the controllers. Once the control plane application is decided the corresponding $\mathrm{Ctr}-\mathrm{Ctr}$ communication may be included in the controller placement optimization by choosing the value of $\lambda$. The optimal performance of the control plane depends both on the latency between switches and controllers ( $\mathrm{Ctr}-\mathrm{Sw})$ and the latency among controllers (Ctr-Ctr). On the other hand, the parameter $\theta$ allows finding better locations for the controllers considering the probability of link failures. A greater failure probability will imply lower values of $\theta$ so that the alternative backup paths have a larger weight in the placement decision. In future research, we plan to consider the capacity of the controllers and links while solving the controller placement and thus analyzing a more practical scenario

\section{ACKNOWLEDGEMENTS}

This research was supported by the Spanish Ministry of Economy and Competitiveness under contract TEC201790034-C2-1-R (ALLIANCE).

\section{REFERENCES}

[1] D. Kreutz, F. M. V. Ramos, P. E. Veríssimo, C. E. Rothenberg, S. Azodolmolky and S. Uhlig, "Software-Defined Networking: A Comprehensive Survey," in Proceedings of the IEEE, vol. 103, no. 1, pp. 14-76, Jan. 2015, doi: 10.1109/JPROC.2014.2371999.

[2] Nunes, B. A. A., Mendonca, M., Nguyen, X. N., Obraczka, K., \& Turletti. A survey of software-defined networking: Past, present, and future of programmable networks. IEEE Communications Surveys and Tutorials, Volume: 16, Issue: 3, Page(s): 1617 - 1634, Third Quarter 2014.

[3] Arya V, Garg N, Khandekar R, Meyerson A, Munagala K, Pandit V. Local search heuristics for k-median and facility location problems. SIAM J Comput. 2004;33(3):544-562.

[4] F. Yeung, "Internet 2: scaling up the backbone for R\&D," in IEEE Internet Computing, vol. 1, no. 2, pp. 36-37, March-April 1997, doi:10.1109/4236.601096.

[5] B. Heller, R. Sherwood, N. McKeown, The controller placement problem, in Proceedings of the First Workshop on Hot Topics in Software Defined Networks (ACM, Helsinki, Finland, 2012), pp. 7-12

[6] G. Wang, Y. Zhao, J. Huang, Q. Duan and J. Li, "A K-means-based network partition algorithm for controller placement in software defined network," 2016 IEEE International Conference on Communications (ICC), Kuala Lumpur, 2016, pp. 1-6, doi: 10.1109/ICC.2016.7511441.

[7] K. S. Sahoo, B. Sahoo, R. Dash and M. Tiwary, "Solving Multicontroller Placement Problem in Software Defined Network," 2016 International Conference on Information Technology (ICIT), Bhubaneswar, 2016, pp. 188-192, doi: 10.1109/ICIT.2016.047.

[8] Y. Fan, Y. Xia, W. Liang, X. Zhang, "Latency-aware reliable controller placements in SDNs," in International Conference on Communications and Networking in China, Springer, 2016, pp. 152-162.

[9] Y. Fan, T. Ouyang, Reliability-aware controller placements in softwaredefined networks, in The 21st IEEE International Conference on HighPerformance Computing and Communications, HPCC 2019, Zhangjiajie, China, 2019.

[10] Singh, AK, Maurya, S, Kumar, N, Srivastava, S. Heuristic approaches for the reliable SDN controller placement problem. Trans Emerging Tel Tech. 2020; 31:e3761.

[11] Gao C, Wang H, Zhu F, Zhai L, Yi S. A particle swarm optimization algorithm for controller placement problem in software defined network. In: Proceedings of the International Conference on Algorithms and Architectures for Parallel Processing (ICA3PP 2015); 2015; Zhangjiajie, China.

[12] Singh AK, Kumar N, Srivastava S. PSO and TLBO based reliable placement of controllers in SDN. Int $\mathbf{J}$ Comput Netw Inf Secur. 2019;11(2):36-42.

[13] Rao RV. Jaya: a simple and new optimization algorithm for solving constrained and unconstrained optimization problems. Int J Ind Eng Comput. 2016;7(1):19-34.

[14] K. S. Sahoo, A. Sarkar, S. K. Mishra, B. Sahoo, D. Puthal, M. S.Obaidat, and B. Sadun, "Metaheuristic solutions for solving controller placement problem in sdn-based wan architecture," 2017.

[15] Kurdman Abdulrahman Rasol, J Domingo-Pascual, Joint Placement Latency Optimization of the Control Plane in 2020 International Symposium on Networks, Computers and Communications (ISNCC), Montreal - Canada, 2020. 